\title{
Enhancing fracture toughness of nanotwinned austenitic steel by thermal annealing
}

\author{
L. Xiong ${ }^{\mathrm{a}}$, Z.S. You ${ }^{\mathrm{a}, \mathrm{b}}$ and L. $\mathrm{Lu}^{\mathrm{a}, *}$ \\ ${ }^{a}$ Shenyang National Laboratory for Materials Science, Institute of Metal Research, \\ Chinese Academy of Sciences, 72 Wenhua Road, Shenyang 110016, People's \\ Republic of China \\ ${ }^{b}$ Herbert Gleiter Institute of Nanoscience, Nanjing University of Science and \\ Technology, 200 Xiaolingwei Street, Nanjing 210094, People's Republic of China \\ * Corresponding author. E-mail addresses: llu@imr.ac.cn
}




\begin{abstract}
The fracture behavior was investigated of a $316 \mathrm{~L}$ austenitic stainless steel with heterogeneously distributed nanotwin bundles, prepared by dynamic plastic deformation and subsequent annealing at different temperatures. The results indicate that the controlled annealing causes a slight decrease in the strength, but remarkably improves the fracture resistance, and therefore enhances the strength-toughness synergy. The toughness enhancement is attributable to the formation of recovered sub-micron-sized and recrystallized micron-sized grains postponing crack nucleation and to the remnant nanotwin bundles arresting crack propagation.
\end{abstract}

Keywords: Dynamic plastic deformation; Austenitic stainless steel; Nanotwins; Fracture toughness; Thermal annealing. 
Austenitic stainless steels (SS) are widely used in engineering applications due to their excellent corrosion resistance and good formability [1]. But their low yield strengths in general limit the applications as energy efficient structural components [2]. Grain refinement by severe plastic deformation has attracted considerable attention to develop high strength steels [3-5]; however, the strength increment by this method is always accompanied with dramatic reduction in ductility and fracture toughness [5-7]. The reduced fracture toughness has an important bearing on the suppression of dislocation activities within the nanoscale tiny grains [7-9], and on the fact that the numerous large angle grain boundaries (GBs) in three-dimensional network can directly act as micro-void nucleation sites and crack extension paths $[10,11]$. Therefore, structural reliability and hence potential engineering applications of nanograined components are still seriously restricted by their limited damage tolerance.

By contrast, two-dimensional coherent twin boundaries (TBs) at the nanometer scale are more resistant to crack nucleation and propagation, while maintaining a significant strengthening capability [12-15]. For instance, by introducing bundles of nanoscale deformation twins into a matrix of nanograins, Qin et al. discovered that the nanotwin bundles could enhance the crack growth resistance by triggering the formation of coarse/deep dimples [16,17]. However, the presence of a large volume fraction of nanograins in the deformed samples still limits the crack initiation toughness, since micro-cracks easily nucleate and coalesce in the nanograined matrix. To solve this problem, controlled thermal treatment to modify the nanograined 
microstructure, which has been recognized to be an effective approach to deal with the strength-ductility trade-off relationship $[18,19]$, can be a feasible strategy.

For the case of austenitic steels, it has been demonstrated that dynamic plastic deformation (DPD) in conjunction with optimized thermal annealing to produce a mixed structure consisting of nanotwinned and recrystallized austenitic grains could bring about a good combination of high strength and large tensile ductility [20,21]. However, there is still a lack of fracture mechanics evaluation on the damage tolerance of this unique material, which is highly required for engineering applications. In this work, the fracture behavior of the nanotwinned austenitic $316 \mathrm{~L}$ SS was investigated, majorly aiming at evaluating the contributions of nanotwin bundles and recrystallized grains to the fracture toughness, and at exploring the underlying strengthening and toughening mechanisms.

The used 316L SS and the DPD treatment in this work are identical to those in Refs. [21,22]. Cylindrical coarse-grained samples, $12 \mathrm{~mm}$ in diameter and $16 \mathrm{~mm}$ in height, were subjected to repeated DPD treatments up to a total true strain of 1.6. The strain is defined as $\varepsilon=\ln \left(h_{\mathrm{i}} / h_{\mathrm{f}}\right)$, where $h_{\mathrm{i}}$ and $h_{\mathrm{f}}$ are the initial and final thickness of the treated sample. In order to further modify the microstructure, the as-DPD disc samples were annealed at $710-730{ }^{\circ} \mathrm{C}$ for $20 \mathrm{~min}$ and then water-quenched. Uniaxial tensile tests were performed on an Instron 5848 micro-tester ( $2 \mathrm{kN}$ load capacity) at a strain rate of $3 \times 10^{-3} \mathrm{~s}^{-1}$ at room temperature, using dog bone-shaped samples with a gage length of $5 \mathrm{~mm}$. A contactless MTS LX300 laser extensometer was used to accurately measure the imposed strain upon loading. 
In order to evaluate the fracture toughness, miniaturized single edge-notched specimens with a thickness of $2 \mathrm{~mm}$, a width of $4 \mathrm{~mm}$, and a span distance of $16 \mathrm{~mm}$ were machined from the as-DPD and thermally treated discs, with the thickness direction corresponding to that of the DPD disc. The crack propagation direction is parallel to the radial direction of the disc, while the propagation plane is normal to the tangential direction. The specimens were first notched to a depth of $\sim 800 \mu \mathrm{m}$ by electrical discharge machining, and then pre-cracked under cyclic bending to an original crack length $a_{0}$ of $\sim 2 \mathrm{~mm}$. The pre-cracked specimens were finally monotonically bent to extend the crack at a constant displacement rate of $0.3 \mathrm{~mm}$ $\min ^{-1}$ on the Instron 5848 micro-tester. During the test, the instantaneous crack length was monitored using the direct current potential drop method. With the synchronously recorded force $P$, load-line displacement $v$ and crack extension $\Delta a$, the elastic-plastic fracture toughness (critical $J$-integral) could be determined from the $J$-integral resistance curves calculated based on the recommendations of the ASTM E1820-11 [23].

The microstructure was characterized by field emission gun scanning electron microscopy (SEM) in a FEI Nova NanoSEM 430 microscope and by transmission electron microscopy (TEM) using a JEOL 2010 microscope operated at $200 \mathrm{kV}$. Electron backscatter diffraction (EBSD) measurements and analysis were performed using the HKL channel 5 software suite. The fracture surfaces were examined by the SEM with secondary electron imaging, and by the Olympus LEXT OLS4000 confocal laser scanning microscope (CLSM) to determine the three-dimensional fracture 
topography.

Fig. 1a shows the typical cross-sectional microstructure of the as-DPD sample, which is spatially heterogeneous and is characterized by rhombic bundles of nanoscale deformation twins embedded in a matrix of nano-sized grains. The nanotwin bundles with longitudinal length ranging from several to tens of micrometers are remnants surviving from the extensive localized shear band deformation in various directions. Closer TEM observations reveal a high density of dislocations accumulated along the TBs (Fig. 1b), characteristic of deformation twins. The statistical results indicate that the nanotwin bundles with a mean twin/matrix thickness of $\sim 16 \mathrm{~nm}$ take up a total volume fraction of $\sim 26 \%$, with the balance being occupied by the nanograins. Most of the nanograins are generated inside the shear bands through the fragmentation and rotation of the twin/matrix lamellae, and hence are slightly elongated with an average transverse size of $\sim 30 \mathrm{~nm}$ and a mean longitudinal size of $\sim 80 \mathrm{~nm}$.

Additional thermal annealing at $710-730{ }^{\circ} \mathrm{C}$ leads to substantial variations in the deformed microstructure. Fig. 1c shows the typical microstructure of the DPD sample annealed at $720{ }^{\circ} \mathrm{C}$ for $20 \mathrm{~min}$. Obviously, nano-sized grains are much less stable due to the high stored energy comparing to nanotwined structures, and hence partial recrystallization preferentially occurs in the nanograined areas to convert some of them to micron-sized recrystallized grains that are visible under the SEM observations. The EBSD measurements (Fig. 1f) reveal the presence of large angle misorientations between the recrystallized grains and the formation of the annealing twins inside. Due 
to the relatively low annealing temperatures, some deformed nano-grains are just recovered and become dislocation-free. These recovered sub-micron-sized grains usually surround the nanotwinned bundles, as shown in closer TEM observation (Fig. 1d). After the thermal annealing, there is no significant change in the volume fraction or the average twin/matrix thickness of the nanotwin bundles, due to their low interface energy and high thermal stability [24]. However, a large fraction of dislocations accumulated at TBs annihilate after the annealing (Fig. 1e). The statistical results of the microstructure for the as-DPD and the annealed DPD samples are summarized in Table 1.

The representative tensile engineering stress-strain curves for the as-DPD and the thermally treated samples are shown in Fig. 2. DPD to $\varepsilon=1.6$ substantially elevates the yield strength $\sigma_{\mathrm{y}}$ up to $1366 \mathrm{MPa}$ and the ultimate tensile strength $\sigma_{\mathrm{uts}}$ to $1416 \mathrm{MPa}$. However, it also dramatically reduces the tensile ductility. After the annealing treatments, the strengths (both $\sigma_{\mathrm{y}}$ and $\sigma_{\mathrm{uts}}$ ) slightly drop due to the reduction in dislocation density and moderate grain coarsening. In return, an obvious increment in ductility is observed. For instance, the uniform elongation increases to $4.2 \%$ for the sample annealed at $720{ }^{\circ} \mathrm{C}$.

Fig. 3a shows the $P-v$ curves for the as-DPD and heat-treated samples obtained from the three-point bending tests. The instantaneous electric resistance $R$ between the two ends of the bending sample was also automatically recorded to measure the crack growth, see the dash lines in Fig. 3a. Based on the $P-v$ curves and the crack extension $\Delta a=a-a_{0}$ inferred from the calibration curves between $R$ and crack length $a$, the 
fracture resistance, $J$-integral, as a function of $\Delta a$ can be computed, as presented in Fig. 3b. All the samples exhibit stable crack growth behavior, and the $J$-integral monotonically increases with $\Delta a$. But with increasing annealing temperature, the slope of $J$-integral resistance curve increases, indicating an enhanced crack propagation resistance for samples annealed at higher temperatures.

According to the $J$-integral resistance curve, the critical fracture toughness $\left(J_{\mathrm{IC}}\right)$ is in general evaluated as its intersection point with the $0.2 \mathrm{~mm}$ offset crack blunting line (, where $\sigma_{\mathrm{Y}}$ is the average value of $\sigma_{\mathrm{y}}$ and $\sigma_{\mathrm{uts}}$ ), as displayed in Fig. $3 \mathrm{~b}$. Then the critical stress-intensity factor $\left(K_{\mathrm{IC}}\right)$ can be calculated from $J_{\mathrm{IC}}$ by using the following relationship:, where $E$ is the Young's modulus and $v$ is the Poisson's ratio, respectively. $J_{\mathrm{IC}}$ and $K_{\mathrm{IC}}$ values of all the tested $316 \mathrm{~L}$ samples are also listed in Table 1, which shows that $K_{\mathrm{IC}}$ of the as-DPD sample is $117 \mathrm{MPa} \mathrm{m}^{1 / 2}$, and increases with increasing annealing temperature. After annealed at $730{ }^{\circ} \mathrm{C}$ for $20 \mathrm{~min}$, the $K_{\mathrm{IC}}$ is 172 $\mathrm{MPa} \mathrm{m}^{1 / 2}$, which is remarkably enhanced compared to that of the as-DPD sample. Note that the $\sigma_{\text {uts }}$ is still as high as $1.1 \mathrm{GPa}$, mildly reduced with respect to that of the as-DPD sample. The above results suggest that a good synergy of strength and fracture toughness can be accomplished by thermo-mechanical treatment involving nanoscale twins, which is superior to other strengthening strategies [6].

To understand the enhanced fracture toughness, the fracture surfaces of the as-DPD and $720{ }^{\circ} \mathrm{C}$ annealed samples were examined by SEM and CLSM, as shown in Fig. 4. SEM observations reveal that the fracture surface of the as-DPD sample exhibits ductile fracture mode, which is evidently composed of two kinds of dimples 
(see Fig. 4a). One is relatively coarse and deep with an average longitudinal length of $\sim 30 \mu \mathrm{m}$; the other is rather equiaxed and fine with a mean diameter of $\sim 5 \mu \mathrm{m}$. While the fine dimples were prominently observed on the fracture surfaces of homogeneous nanocrystalline and ultrafine grained materials $[6,25,26]$, the coarse dimples have only been identified in the heterogeneous structure with embedded nanotwin bundles $[16,17]$. The coarse/deep dimples constitute an area fraction of $\sim 26 \%$, close to the volume fraction of the embedded nanotwins, manifesting that their formation is intimately connected with the presence of the nanotwin bundles. The CLSM 3D morphology in Fig. 4b reveals the depth of different dimples, and the relative peak-valley height of the selected area can be as large as $\sim 80 \mu \mathrm{m}$.

Fig. 4c shows the typical fracture surface of the $720{ }^{\circ} \mathrm{C}$ annealed sample, which is also composed of two kinds of dimples. And apparently, the applied annealing treatment increases the dimple sizes, especially for the fine ones. The average size of the fine dimples $(\sim 9 \mu \mathrm{m})$ is approximately two times as large as that of the as-DPD sample, while the average longitudinal length of the coarse dimples is slightly increased to $\sim 40 \mu \mathrm{m}$. The area fraction of coarse/deep dimples is $\sim 29 \%$, nearly the same as that of the as-DPD sample, in consistent with the remaining nanotwins. The CLSM observations (Fig. 4d) indicate the fracture surface becomes even more rough and the relative peak-valley height of the surface increases to about $110 \mu \mathrm{m}$. Table 2 summarizes the statistical results of the dimple sizes and area fractions for the as-DPD and annealed DPD samples. The area fractions of the two kinds of dimples remain unchanged upon the mediate temperature annealing at $710-730{ }^{\circ} \mathrm{C}$, but increasing the 
annealing temperature slightly elevates their sizes.

Combining the results of tensile tests and fracture toughness measurements for the thermally annealed DPD 316L SS (Table 1), it is suggestive that the incorporation of nanoscale twins as appealing strengthening and toughening agents in bulk metals and alloys is highly practical under optimized deformation conditions (to stimulate deformation twinning) in conjunction with suitable heat treatment. This feasibility originates from several special features of the nanoscale twins, including a good combination of strength and deformability arising from TB/dislocation interactions $[12,27,28]$, strong fracture resistance of coherent TBs $[14,15,29]$, and superior thermal stability [24].

The as-DPD sample simultaneously possesses a high strength and a strong fracture toughness, which provides a favorable starting point for subsequent thermal treatment. The high strength arises from the presence of nano-sized grains and twin lamellae, while the fracture toughness is closely associated with the nanotwin bundles. Qin et al. revealed that the embedded nanotwin bundles are responsible for forming coarse/deep dimples (like those in Fig. 4a) and thus increase the resistance to the crack propagation [17]. However, in the mixed structure of nanotwins and nanograins, the cracks tend to nucleate at the abundant GBs or triple junctions, and then extend through the nanograin matrix easily [30]. The nanograin matrix with limited deformability is still the weak link that constrains further enhancement in fracture resistance of the deformed sample [30].

Controlled heat treatment effectively modifies the microstructure of the nanograin 
matrix by increasing the grain size and eliminating the stored extrinsic dislocations. More importantly, the GB structures are also altered intrinsically by recrystallization or GB migration during annealing [31,32]. It is believed that the nucleation of GB crack is caused by the strain incompatibility between neighbor grains, which is largely dependent on the GB characteristics [10]. Thermal treatment would reduce the volume fraction of GBs and some potential crack nucleation sites. Furthermore, the clearing up of the stored dislocations would increase the critical strain for crack nucleation. These analyses are experimentally proved by the detection of much larger dimples on the fracture surface of the heat treated samples (Figs. 4c-d). The increment in dimple size elevates the irreversible plastic energy dissipated during crack propagation. Taking account of the large volume fraction $(\sim 70 \%)$, the enhanced facture toughness of the annealed DPD samples can be attributed to the formation of recovered and recrystallized microstructures.

Another advantage of the annealed samples arises from the annealed deformation twins, which not only strengthen the material, but also to certain extent contribute to the improved fracture toughness. Due to the high thermal stability, the heat treatment does not substantially change the volume fraction or the thickness of the twins. Therefore, it is anticipated that the nanotwin bundles almost retain their contribution to the total strength after annealing, as confirmed by previous tensile tests [22]. The strength contributed by the nanotwin bundles is estimated to be as high as $420 \mathrm{MPa}$ ( $40 \%$ ) for the $730{ }^{\circ} \mathrm{C}$ annealed sample, taking account of their strength and volume fraction. Besides the strengthening, the additional toughening arising from the 
annealed nanotwins should not be neglected either. The thermal annealing removes some accumulated dislocations at TBs and hence recovers the deformability of nanotwins, which is beneficial for accommodating the plastic incompatibility between the nanotwins and the surrounding recrystallized grains. Furthermore, a strain gradient was observed to develop at the transitional area from the nanotwin bundle to the recrystallized grains, which stimulates deformation twinning in the recrystallized grains immediately near the bundle [33]. The enhanced plastic deformation of the nanotwin bundle and the neighboring recrystallized grains increases the energy dissipation for generating the coarse dimples that are much larger than those formed during the fracture of the as-DPD sample (Table 2).

In summary, the fracture behavior is investigated of the nanostructured $316 \mathrm{~L} \mathrm{SS}$ with a mixed structure of nanotwin bundles embedded in the matrix of deformed nanograins and of annealed submicron grains. The microstructural modulation involving recovery and controlled recrystallization of nanograins leads to an enhanced strength-fracture toughness synergy. The improved toughness is predominantly attributed to the recovered or partially recrystallized grains that suppress the crack nucleation leading to much larger dimple sizes and to the annealed nanotwin bundles that impede the crack propagation.

\section{Acknowledgments}

The authors acknowledge financial support from the National Basic Research Program of China (973 Program, 2012CB932202), the National Science Foundation 
of China (Grant Nos. 51420105001, 51371171, 51471172 and 51401211). LL thanks the financial support of the "Hundreds of Talents Project" by the Chinese Academy of Sciences.

\section{References}

[1] P. Marshall, Austneite Stainless Steel: Microstructure and Mechanical Properties, Elsevier Applied Science Publishers, New York, 1984.

[2] M.F. Ashby, Materials Selection in Mechanical Design, fourth ed., Butterworth-Heinemann, New York, 2010.

[3] B.P. Kashyap, K. Tangri, Acta Mater. 45 (1997) 2383-2395.

[4] I. Ucok, T. Ando, N.J. Grant, Mater. Sci. Eng. A 133 (1991) 284-287.

[5] X.H. Chen, J. Lu, L. Lu, K. Lu, Scr. Mater. 52 (2005) 1039-1044.

[6] A. Hohenwarter, R. Pippan, Phil. Trans. R. Soc. A 373 (2015) 20140366.

[7] M.A. Meyers, A. Mishra, D.J. Benson, Prog. Mater. Sci. 51 (2006) 427-556.

[8] M. Dao, L. Lu, R.J. Asaro, J.T.M. De Hosson, E. Ma, Acta Mater. 55 (2007) $4041-4065$.

[9] K.S. Kumar, H. Van Swygenhoven, S. Suresh, Acta Mater. 51 (2003) 5743-5774.

[10] K.S. Kumar, S. Suresh, M.F. Chisholm, J.A. Horton, P. Wang, Acta Mater. 51 (2003) 387-405.

[11] I.A. Ovid"ko, A.G. Sheinerman, Acta Mater. 52 (2004) 1201-1209.

[12] K. Lu, L. Lu, S. Suresh, Science 324 (2009) 349-352.

[13] A. Singh, L. Tang, M. Dao, L. Lu, S. Suresh, Acta Mater. 59 (2011) 2437-2446. 
[14] S.-W. Kim, X. Li, H. Gao, S. Kumar, Acta Mater. 60 (2012) 2959-2972.

[15] Z. Zeng, X. Li, L. Lu, T. Zhu, Acta Mater. 98 (2015) 313-317.

[16] E.W. Qin, L. Lu, N.R. Tao, K. Lu, Scr. Mater. 60 (2009) 539-542.

[17] E.W. Qin, L. Lu, N.R. Tao, J. Tan, K. Lu, Acta Mater. 57 (2009) 6215-6225.

[18] N. Tsuji, Y. Ito , Y. Saito , Y. Minamino, Scr. Mater. 47 (2002) 893-899.

[19] Y.S. Li, Y. Zhang, N.R. Tao, K. Lu, Scr. Mater. 59 (2008) 475-478.

[20] K. Lu, F.K. Yan, H.T. Wang, N.R. Tao, Scr. Mater. 66 (2012) 878-883.

[21] F.K. Yan, G.Z. Liu, N.R. Tao, K. Lu, Acta Mater. 60 (2012) 1059-1071.

[22] F.K. Yan, N.R. Tao, K. Lu, Scr. Mater. 84-85 (2014) 31-34.

[23] ASTM E1820-11, Standard Test Method for Measurement of Fracture Toughness, ASTM International, West Conshohocken, PA, 2011.

[24] X. Zhang, A. Misra, Scr. Mater. 66 (2012) 860-865.

[25] H. Li, F. Ebrahimi, Acta Mater. 54 (2006) 2877-2886.

[26] F. Dallatorre, P. Spatig, R. Schaublin, M. Victoria, Acta Mater. 53 (2005) 2337-2349.

[27] L. Lu, Z.S. You, K. Lu, Scr. Mater. 66 (2012) 837-842.

[28] Z. You, X. Li, L. Gui, Q. Lu, T. Zhu, H. Gao, L. Lu, Acta Mater. 61 (2013) 217-227.

[29] Z.W. Shan, L. Lu, A.M. Minor, E.A. Stach, S.X. Mao, JOM 60 (2008) 71-74.

[30] T. Hanlon, Y.N. Kwon, S. Suresh, Scr. Mater. 49 (2003) 675-680.

[31] N. Kamikawa, X. Huang, N. Tsuji, Hans, Acta Mater. 57 (2009) 4198-4208.

[32] A.Belyakov, T. Sakai, H. Miura, R. Kaibyshev, Tsuza, Acta Mater. 50 (2002) 
$1547-1557$.

[33] F.K. Yan, N.R. Tao, F. Archie, I. Gutierrez-Urrutia, D. Raabe, K. Lu, Acta Mater. $81(2014) 487-500$. 


\section{Figure captions}

Figure 1. (a) Cross-sectional microstructure of the as-DPD 316L SS, showing the nanotwin bundles embedded in a nanograin matrix; (b) closer TEM observation on the deformation twins with a high density of accumulated dislocations; (c) SEM image of the cross-sectional microstructure of the $720{ }^{\circ} \mathrm{C}$ annealed DPD sample, showing some recrystallized micro-sized grains; (d) closer TEM observations of the unresolved areas in (c), displaying the nanotwin bundles surrounded by equiaxed sub-micron grains; (e) TEM image of annealed nanotwins with a relatively low density of dislocations; (f) EBSD map of the selected area in (c), indicating the presence of high angle grain boundaries and annealing twins in the recrystallized grains.

Figure 2. Tensile engineering stress-strain curves for the as-DPD and annealed DPD 316L SS, and the coarse-grained (CG) counterpart for comparison.

Figure 3. (a) Typical curves of $P$ and $R$ versus $v$ for the bending tests of the as-DPD sample and the DPD samples annealed at different temperatures; (b) corresponding $J$-integral resistance curves calculated from the data in (a).

Figure 4. SEM observations on the fracture surface of (a) as-DPD sample and (c) 720 ${ }^{\circ} \mathrm{C}$ annealed DPD sample; (b) and (d), three-dimensional CLSM observations on the fracture surface of the same areas in (a) and (c), respectively. The color bars in the right indicate the relative dimple depth. 


\section{Table Captions}

Table 1. Microstructure, tensile properties, and fracture toughness of the as-DPD 316L SS and DPD samples annealed at different temperatures.

\begin{tabular}{lcccccccc}
\hline \multicolumn{1}{c}{ Sample } & $V_{\mathrm{NT}}$ & $V_{\mathrm{G}}$ & $d$ & $\sigma_{\mathrm{y}}$ & $\sigma_{\mathrm{uts}}$ & $\varepsilon_{\mathrm{f}}$ & $J_{\mathrm{IC}}$ & $K_{\mathrm{IC}}$ \\
& $\%$ & $\%$ & $\mathrm{~nm}$ & $\mathrm{MPa}$ & $\mathrm{MPa}$ & $\%$ & $\mathrm{~kJ} \mathrm{~m}^{-2}$ & $\mathrm{MPa} \mathrm{m}^{1 / 2}$ \\
\hline $\mathrm{DPD}$ & $26 \pm 5$ & - & 33 & $1366 \pm 49$ & $1416 \pm 50$ & $8 \pm 1$ & $63 \pm 2$ & $117 \pm 1$ \\
$\mathrm{DPD}+710^{\circ} \mathrm{C}$ annealed & $28 \pm 2$ & $72 \pm 2$ & 150 & $1144 \pm 39$ & $1294 \pm 56$ & $13 \pm 2$ & $88 \pm 5$ & $136 \pm 4$ \\
DPD $+720^{\circ} \mathrm{C}$ annealed & $23 \pm 6$ & $77 \pm 6$ & 230 & $1113 \pm 55$ & $1222 \pm 8$ & $14 \pm 1$ & $108 \pm 12$ & $151 \pm 8$ \\
DPD $+730^{\circ} \mathrm{C}$ annealed & $21 \pm 2$ & $79 \pm 2$ & 550 & $1020 \pm 15$ & $1115 \pm 15$ & $18 \pm 1$ & $140 \pm 6$ & $172 \pm 5$ \\
\hline
\end{tabular}

$V_{\mathrm{NT}}$, volume fraction of nanotwin bundles; $V_{\mathrm{G}}$, volume fraction of recovered or recrystallized grains; $d$, average grain size; $\sigma_{\mathrm{y}}$, yield strength; $\sigma_{\mathrm{uts}}$, ultimate tensile strength; $\varepsilon_{\mathrm{f}}$, elongation to failure; $J_{\mathrm{IC}}$, critical $J$-integral; $K_{\mathrm{IC}}$, critical stress intensity factor calculated from $J_{\mathrm{IC}}$.

Table 2. Statistics on the sizes $(W)$ and the volume fractions $(V)$ of the two kinds of dimples for the as-DPD and DPD sample annealed at different temperatures.

\begin{tabular}{lllll}
\hline \multirow{2}{*}{ Sample } & \multicolumn{2}{c}{ Coarse dimples } & \multicolumn{2}{c}{ Fine dimples } \\
\cline { 2 - 5 } & $W(\mu \mathrm{m})$ & $V(\%)$ & $W(\mu \mathrm{m})$ & $V(\%)$ \\
\hline $\mathrm{DPD}$ & 31 & $26 \pm 3$ & 5 & $74 \pm 3$ \\
$\mathrm{DPD}+710^{\circ} \mathrm{C}$ annealed & 44 & $31 \pm 3$ & 8 & $69 \pm 3$ \\
DPD $+720{ }^{\circ} \mathrm{C}$ annealed & 40 & $29 \pm 5$ & 9 & $71 \pm 5$ \\
DPD $+730{ }^{\circ} \mathrm{C}$ annealed & 41 & $32 \pm 7$ & 9 & $68 \pm 7$ \\
\hline
\end{tabular}



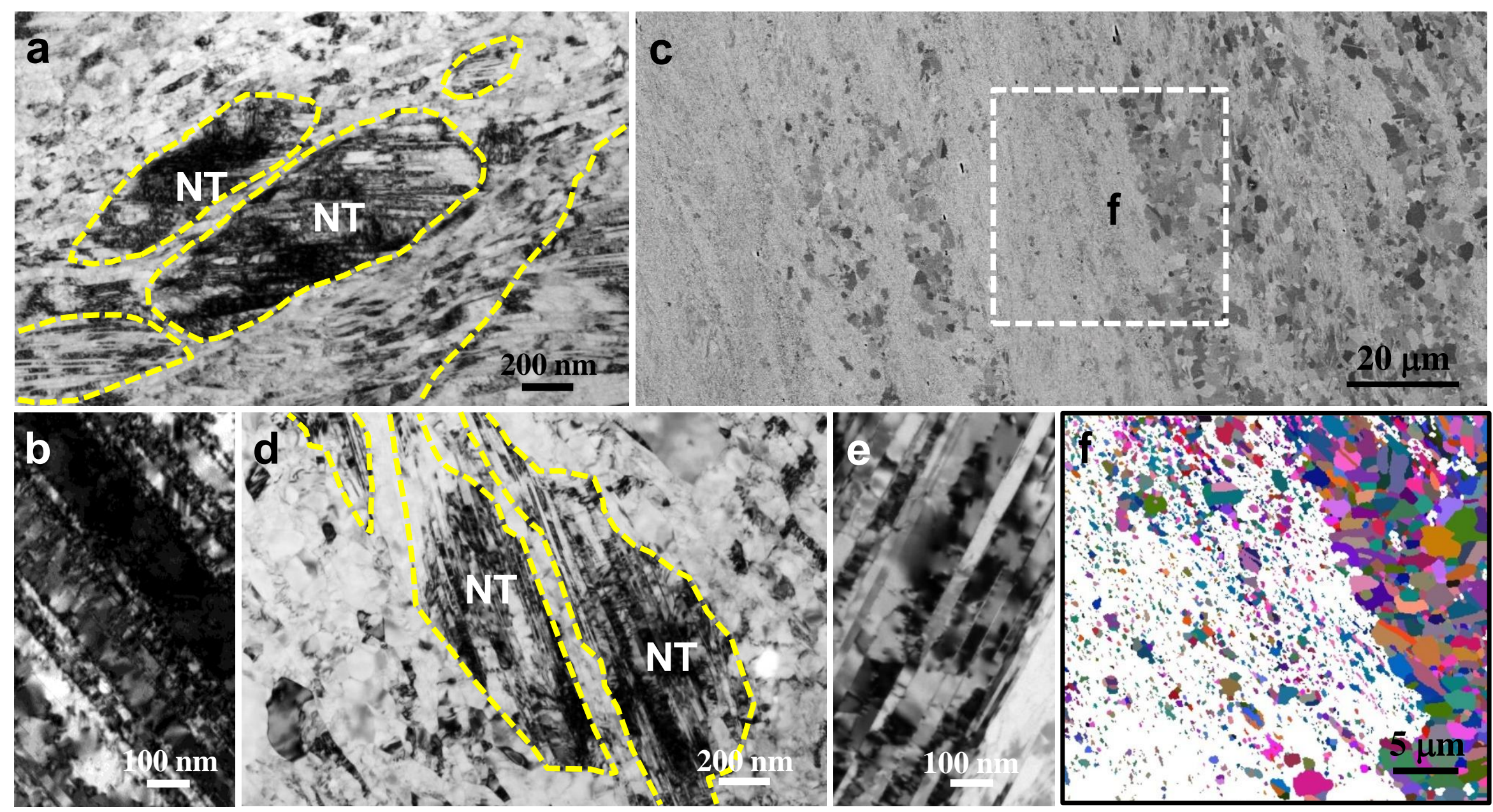

Fig. 1 /Xiong et al 


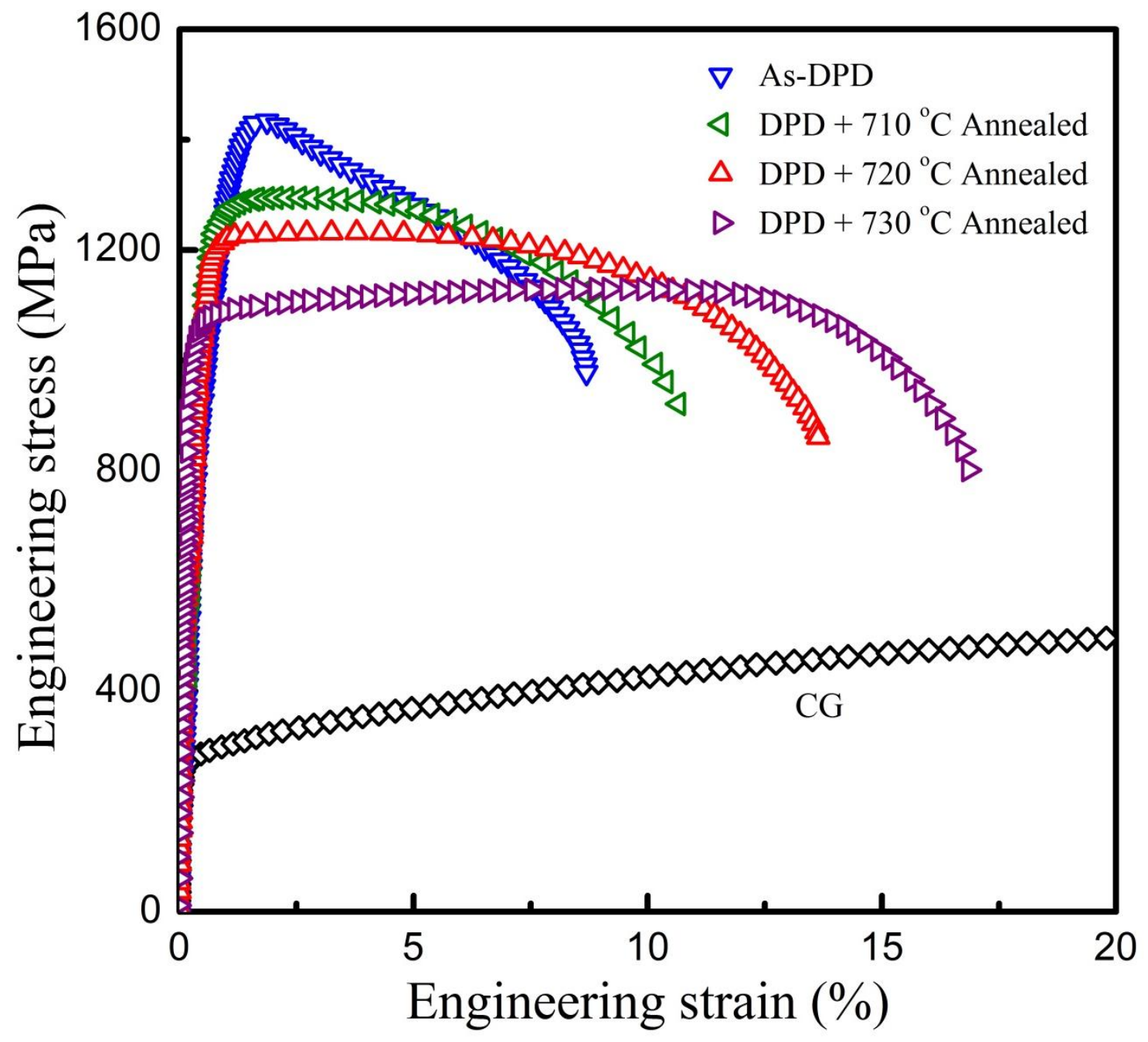

Fig. 2 /Xiong et al 


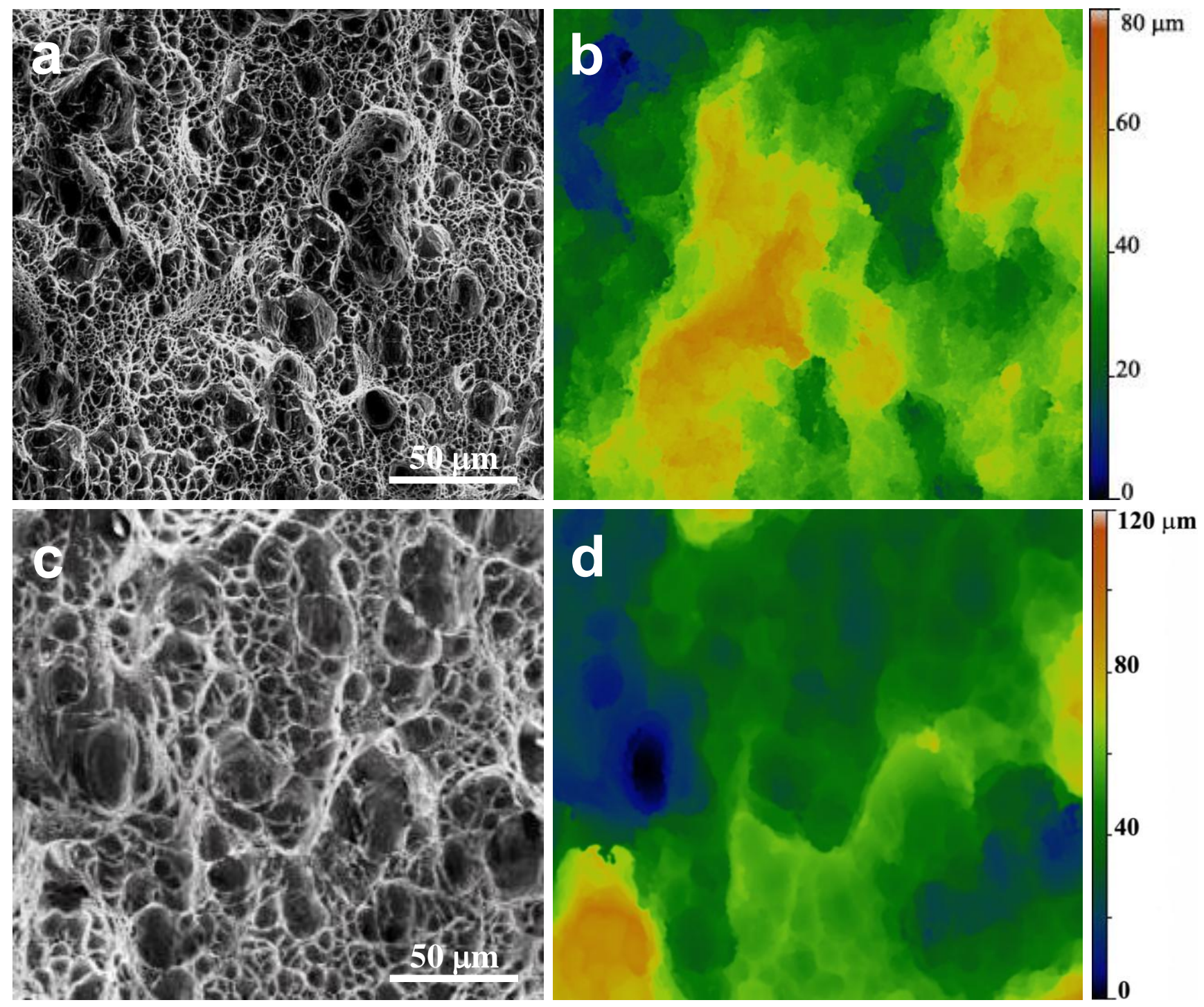

Fig. 4 /Xiong et al 

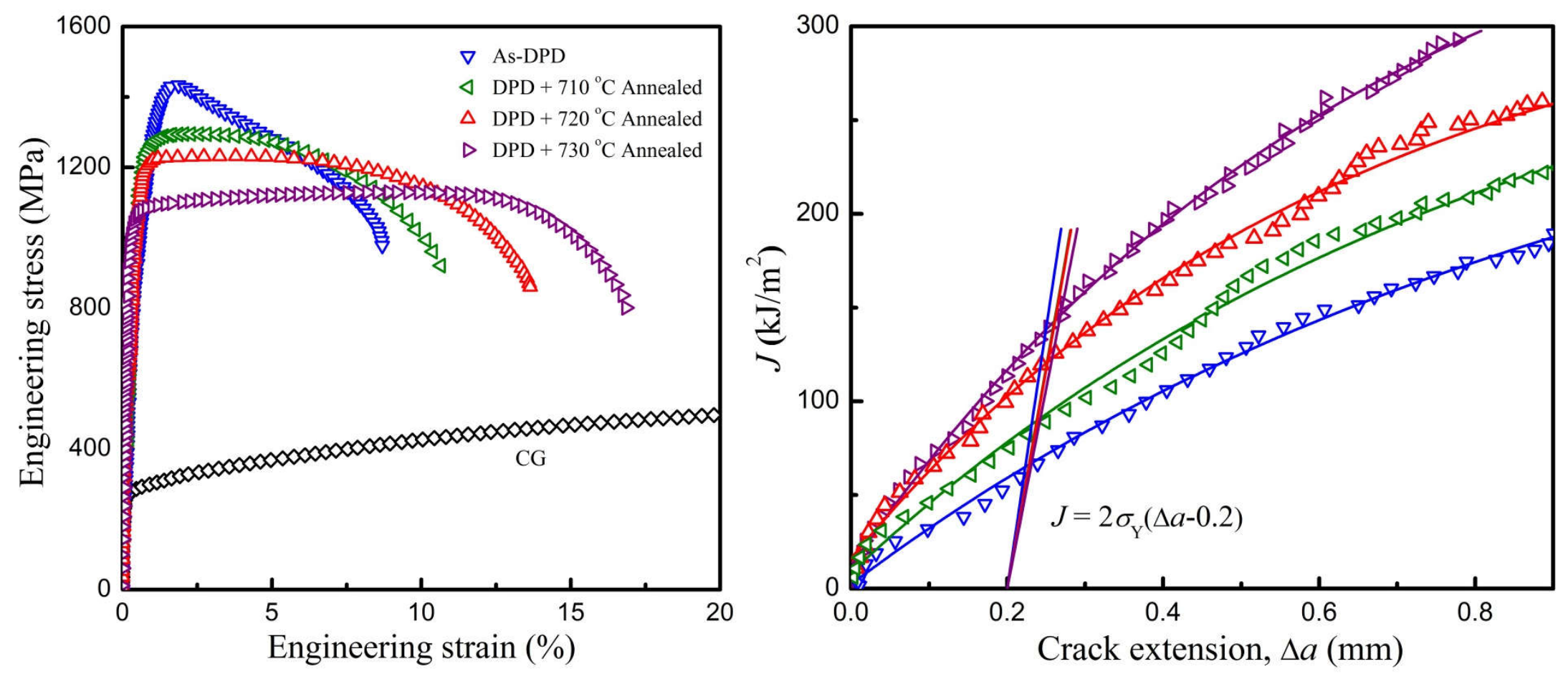

Graphical abstract 